\title{
E-well: Multi-sectoral development of rural areas Project proposal
}

\author{
Antoine Geissbuhler \& Ousmane Ly \\ Medical Informatics Service, Geneva University Hospitals, Geneva, Switzerland; Mali \\ telemedicine network coordinator, REIMICOM, Bamako, Mali
}

antoine.geissbuhler@houge.ch; oussoulv@keneva.net

Abstract: $\quad$ Key aspects of the "e-well"-project concern the evaluation of the impact and sustainability of integrated, multi-sectoral approaches to the development of rural areas in the least advanced countries. The development approaches include, but are note limited to, the usage of multi-purpose tele-centres and the formalisation and publication of local, collective knowledge. Aim is also a reduction of the Digital Divide, particularly obvious in rural areas of developing countries. Focus is on sustainability by fostering simultaneous development activities in multiple sectors (education, health, economy, culture) based on the assessment of local needs.

Key words: developing countries, Digital Divide, Internet access, rural areas, sustainable development, tele-medicine 


\section{INTRODUCTION}

ICT has the potential to improve the quality and efficiency of cooperation and development efforts. However, the risk of ICT-enabled development projects is to further the digital divide between urban and rural areas. It is therefore crucial to involve rural areas early in these efforts, to make sure that these specific needs are addressed in national projects.

In our experience one of the key challenges in the deployment of telemedicine applications in rural Mali, as a component of the national telemedicine network (http://www.sim.hcuge.ch/telemed.html; http://www.keneya.net), is the economic sustainability of such technologies. Sustainability can be improved by fostering simultaneous development activities in multiple sectors (education, health, economy, culture), enabled by an internet-connected tele-centre, the "e-Well". This requires a significant effort, geographically-focused and involving most of the stakeholders of the community, in order to reach a significant increase in development, compatible with long-term sustainability of the process and results.

\section{THE PROJECT}

Based on an assessment of needs by the local authorities, a four-year, multi-sectoral development project has been designed for the community of Dimmbal, a community of 24 villages and 30.000 inhabitants (http://www.dimmbal.ch). Activities include: capacity building for local project coordination, implementation of a tele-centre, development of traditional medicine and its integration in the practice of the rural hospital, development of telemedicine activities with Bamako, valorisation of local history and culture, development of a museum with archaeological findings, revitalization of traditions including the Sacred Wood, and development of the local industry and forestry. The budget for this development project is estimated at $€ 600.000$, over four years, from 2004 to 2007 .

\section{SUCCESS FACTORS}

It is likely that success factors and obstacles in such projects will be educative to similar projects in other settings. The goal of the "e-well » project is to run several development projects in different rural settings in developing countries, and to evaluate, compare and share results in order to learn collectively from the various experiments. The expected outcome is a better understanding of the potential, success factors, impact and 
sustainability, of integrated, multi-sectoral approaches to the development of rural areas in different settings.

The "e-Well" project plans include 6 different sites with four-year development plans, and various coordination, evaluation and sharing activities between the local coordinators of each site. The project is designed to run over 7 years (2004-2010), for a total budget of $€ 6.500 .000$, under the coordination of the AGENTIS, a UNITAR agency dedicated to exploit the potential of information and communication technologies for development and social initiatives.

\section{BIOGRAPHY}

Antoine Geissbuhler is a Professor of Medical Informatics at Geneva University School of Medicine, and Director of the Division of the Medical Informatics at Geneva University Hospitals.

A Philips European Young Scientist first award laureate, he graduated from Geneva University School of Medicine in 1991 and also received his doctorate there. He was trained in internal medicine at Geneva University Hospitals. After a post-doctoral fellowship in medical informatics at the University of Pittsburgh and Vanderbilt University, he became associate professor of biomedical informatics and vice-chairman of the Division of Biomedical Informatics at Vanderbilt University Medical Center, under the mentorship of Prof. Randolph Miller and Prof. William Stead, working primarily on the development of clinical information systems and knowledge-management tools. In 1999, he returned to Geneva to head the Division of Medical Informatics in Geneva University Hospitals and School of Medicine.

His current research focuses on the development of innovative computer-based tools for improving the quality and efficiency of care processes, at the local level of the hospital, the regional level of a community healthcare informatics network, and at the global level with the development of a south-south telemedicine network in Western Africa.

Ousmane Ly is the Executive Coordinator of "Keneya Blown", the technical structure of Mali Network of Information and Medical Telecommunication (REIMICOM). Dr. Ly has a PhD in Medicine and a BSc in Biological Sciences. Currently, he is preparing his Post Medical Computing University Certificate in the University of Geneva. Mr Ly is also a member of the ATAC (African Technical Advisory Committee / United Nations Economic Commission for Africa, ECA). 